\title{
Modelo teórico para el proceso docente en la asignatura de Administración de la Producción III de la carrera de Administración de Empresas
}

\author{
Theoretical model for the teaching process in the subject of Administration \\ of Production III of the career of Administration of Companies
}

\author{
Iver Clive Córdova Ramos \\ iccora@yahoo.com.ar; iccora2@gmail.com \\ Universidad Tomas Frías, Potosí, Bolivia
}

\section{RESUMEN}

El presente artículo tiene la finalidad de dar a conocer los detalles que dieron pie al desarrollo de esta investigación desde una perspectiva analítica, interpretativa y crítica, manteniendo la propósito de contribuir al mejoramiento de las Formas de Organización del Proceso Docente Educativo en la asignatura de Administración de la Producción III. El método empleado fue mediante un diagnostico respectivo, se realizó una indagación censal. Para un mejor análisis se formularon y aplicaron los instrumentos a un cierto porcentaje de estudiantes que ya cursaron la materia y a estudiantes que aún continúan llevando la misma, para lo cual se utilizó muestreo para poblaciones finitas. Los resultados del diagnóstico efectuado muestran los vacíos encontrados en la actual forma de organización de la clase magistral, concentrándose más en la exposición del docente y no así en la participación activa de los estudiantes, lo cual influye en el desarrollo de las clases en la apropiación de contenidos. Se puede concluir que el modelo holístico motivacional propuesto, dirigido a mejorar el proceso enseñanza y aprendizaje en la asignatura, aporta una metodología al docente para motivar a los estudiantes, que es viable.

Palabras clave: Modelo teórico; modelo holístico motivacional; proceso docente; administración de empresas

\section{ABSTRACT}

This article has the purpose of making known the details that gave rise to the development of this research from an analytical, interpretative and critical perspective, maintaining the purpose of contributing to the improvement of the Forms of Organization of the Educational Teaching Process in the Administration subject of Production III. The method used was through a respective diagnosis, a sensory inquiry was made. For a better analysis, the instruments were formulated and applied to a certain percentage of students who have already taken the subject and to students who are still carrying it, for which sampling was used for finite populations. The results of the diagnosis made show the gaps found in the current form of organization of the master class, focusing more on teacher exposure and not on the active participation of students, which influences the development of classes in the appropriation of contents. It can be concluded that the proposed holistic motivational model, aimed at improving the teaching and learning process in the subject, provides a methodology for the teacher to motivate students, which is viable. provides a methodology for the teacher to motivate students, which is viable.

Key words: Theoretical model; holistic motivational model; teaching process; business administration 


\section{INTRODUCCIÓN}

La pertinencia de la educación superior se considera primordialmente en su cometido en la sociedad, en función a la enseñanza y aprendizaje además, dentro del campo de la investigación que requieren los estudiantes, para que estos se mantengan paulatinamente en constante actualización de sus conocimientos y en la adquisición de conocimientos nuevos.

Dada la a la apertura a los nuevos modelos de los procesos de enseñanza y aprendizaje en la educación superior, resulta indispensable establecer propuestas que fomenten la capacidad intelectual de las personas que acuden a las aulas universitarias y que través de la motivación holística los estudiantes puedan asimilar los contenidos de manera eficaz.

Todo proceso de enseñanza $\mathrm{y}$ aprendizaje es un sistema, en el que se da la integración de la instrucción y la educación bajo determinadas condiciones sociohistóricas como una expresión integrada de la diversidad de influencias educativas dentro del proceso enseñanza y aprendizaje.

En la carrera de Administración de Empresas de la Universidad Tomas Fría en Bolivia, las bases, fundamentos teóricos, metodológicos, señalan estar acorde a las exigencias y contar con múltiples medidas para acrecentar la superación de la educación superior, para que el futuro profesional sea acreedor y transformador de su propio medio.

La carrera de Administración de Empresas cuenta en la malla curricular con la asignatura de Administración de la Producción III, misma que tiene una connotación trascendental ya que su objetivo es transmitir el cúmulo de conocimientos sobre el manejo y aplicación racional de los recursos de las empresas.

El diagnóstico realizado en la institución para llevar a cabo el proceso investigativo pudo determinar que los estudiantes manifestaban que la asignatura en cuestión no era de su agrado por ser una materia repetitiva y memorística, lo que provoca baja motivación entre ellos, por ser una materia memorística y no analítica, además, de no favorecer al estudiante para que este se apropie de los conocimientos, lo cual afecta a la eficiencia en el aprendizaje de las clases impartidas, manifestaban que no se resuelven casos reales con respecto a empresas en Bolivia, lo que ocasiona que el estudiante no tenga una estrecha relación con el manejo y la solución de conflictos a nivel laboral.

Lo que pone a las clases magistrales como agotadoras para el estudiante puestos que estos debe tomar apuntes y provoca la escasa participación del estudiante; el docente lleva toda la iniciativa, el estudiante se mantiene pasivo y receptivo; el docente utiliza la clase magistral y no se apoya en recursos y medios audiovisuales lo que provoca tedio al estudiante; la clase magistral no motiva al estudiante por lo que los estudiantes no amplían la materia posteriormente; esta forma de organización de clase magistral no permite un adecuado control del aprendizaje de los temas desarrollados por el docente; y los estudiantes de la asignatura no tienen conciencia de la magnitud, importancia y trascendencia acerca del equipamiento de la planta y factores de mejoramiento de la productividad para su vida laboral y lo que conllevaría promover el desarrollo empresarial.

Es por ello, que el propósito de la investigación fue proponer un modelo teórico de la Forma de Organización Holístico Motivacional para optimizar el proceso Enseñanza y Aprendizaje en la asignatura de Administración de la Producción III de la carrera de Administración de Empresas de la Universidad Autónoma "Tomas Frías”. 
Para dar una adecuada respuesta a las interrogantes formuladas fue importante realizar las siguientes tareas de investigación: Realizar una investigación bibliográfica de las diferentes teorías en relación con las Formas de Organización del proceso Docente Educativo; diagnosticar la situación actual de la forma de organización de clase de la asignatura Administración de la Producción III.; elaborar el modelo teórico de la forma de organización holística motivacional para mejorar el proceso enseñanza aprendizaje de la asignatura Administración de la Producción III de la carrera de Administración de Empresas; y Valoración de la viabilidad del modelo planteado.

\section{MÉTODO}

Para llevar a cabo el proceso investigativo se sistematizó y analizaron documentos que permitieron efectuar una contrastación de los conceptos y definiciones de los distintos autores recogidos a través de la investigación bibliográfica; el método inductivo - deductivo, inicialmente el método deductivo en la información bibliográfica, hasta llegar a la identificación del tema específico de Investigación, posteriormente el método inductivo se utilizó para desarrollar el objeto de estudio. Lo cual fue útil para concluir la información necesaria e inducirla al objeto de estudio.

Igualmente el método histórico - lógico, que permitió obtener información sobre antecedentes de creación del objeto de estudio. Y por último se usó el método de modelación, se utilizó después de haber realizado el marco teórico y el diagnóstico para asumir una propuesta teórica referente a la Forma de Organización Holística Motivacional.

Asimismo, las técnicas utilizadas fueron la técnica del fichaje que ayudó a desarrollar el marco teórico, con base al cual se podía obtener una solución tentativa al problema, la técnica de la encuesta en la elaboración y aplicación de los cuestionarios a los estudiantes para obtener información sobre el conocimiento que tienen los mismos sobre las formas de organización del proceso docente educativo. También se aplicó la entrevista a algunos docentes conocedores de la materia con el fin de obtener información de primera fuente respecto al tema de investigación.

\section{Formas de organización docente educativo}

Es fundamental identificar la tarea de la didáctica en la estructura de los distintos componentes que caracterizan el proceso, es decir, el contenido, el método, medios y la evaluación, de tal manera se pueda cumplir con los objetivos pero fundamentalmente con el encargo social.

Para Álvarez (2006) la forma, es la organización, el orden que adopta el proceso para alcanzar el objetivo, en el que destaca primero la relación profesor estudiante. Esta conceptualización de Álvarez hace hincapié en la relación que debe existir entre el profesor y el estudiante o los estudiantes, pudiendo ser esta de carácter grupal o individual. Esta conceptualización muestra un panorama más amplio de lo que significa la forma, es decir, la conceptualiza como parte del Proceso Docente Educativo siendo la misma un componente que desmarca la organización para alcanzar lo planteado.

Sin embargo, para Ruíz (2008) la Forma de Organizativa del PDE es la estructuración de la actividad del profesor y de los estudiantes con el fin de lograr, de la manera más eficiente, los objetivos de los planes y programas de estudio, mediante la utilización de métodos y medios de enseñanza $y$ aprendizaje que contribuyan al mejor desarrollo del proceso y a la apropiación, por 
parte de los estudiantes, de los conocimientos y habilidades inherentes al objeto del trabajo del profesional.

Por último, conocer los resultados, que se van obteniendo, resulta posible mediante la implementación de un importante componente del proceso: la evaluación. Ésta parte de la definición misma de los objetivos y concluye con la determinación del grado de eficiencia del proceso, en virtud de alcanzar los objetivos propuestos para el logro de un aprendizaje desarrollador.

\section{RESULTADOS Y DISCUSIÓN Modelo teórico de forma de organización holística motivacional para el proceso docente educativo}

A continuación se muestra el modelo que se creó producto de la labor investigativa en la Universidad Tomas Frías el cual ofrecerá una nueva manera de analizar el Proceso Docente Educativo, adecuados a los procesos sociales, de naturaleza consciente, holística y dialéctica. La utilidad del modelo radica en su capacidad para estimular conscientemente al individuo, con el objetivo de satisfacer las necesidades educativas; pues de ellas depende el sentido que se le conceda al proceso de aprendizaje.

En la figura 1 muestra los elementos que se tomaron para el establecimiento del modelo teórico propuesto, en donde se explica cada uno de ellos de la misma manera a responder las interrogantes de "para que, qué y el con que", teniendo ya establecido el objetivo de la materia "Introducir al estudiante hacia un desarrollo intelectual y hábitos de investigación en el manejo y aplicación racional de los recursos de las empresas, con el objeto de mejorar el nivel de productividad de las mismas y propiciar de esta manera, su crecimiento. Se procedió a desarrollar el modelo.

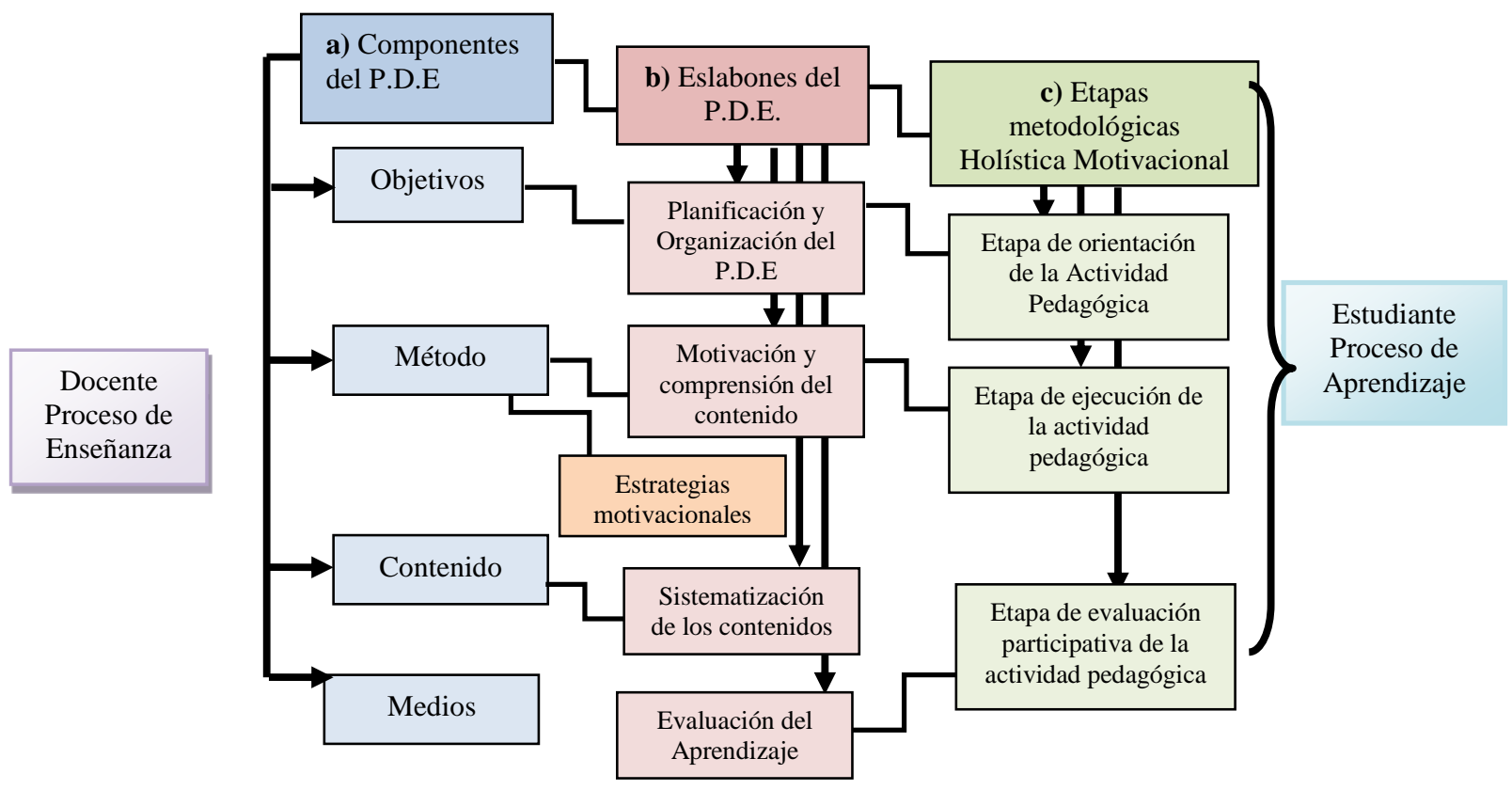

Figura 1. Modelo teórico de forma de organización holística motivacional para el proceso docente educativo 
El modelo planteado fue diseñado por la autora con base a las teorías consultadas, esto con la finalidad de mejorar la comprensión dentro de la propuesta, en la figura siguiente se muestra el desarrollo del modelo propuesto.

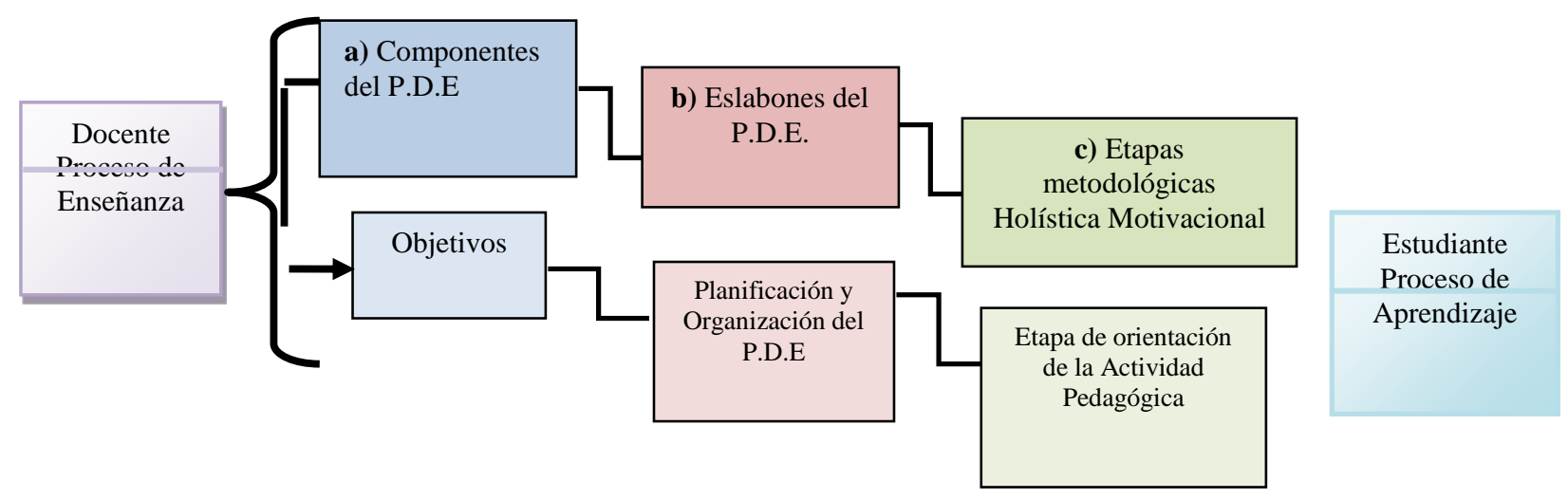

Figura 2. Desarrollo del modelo propuesto

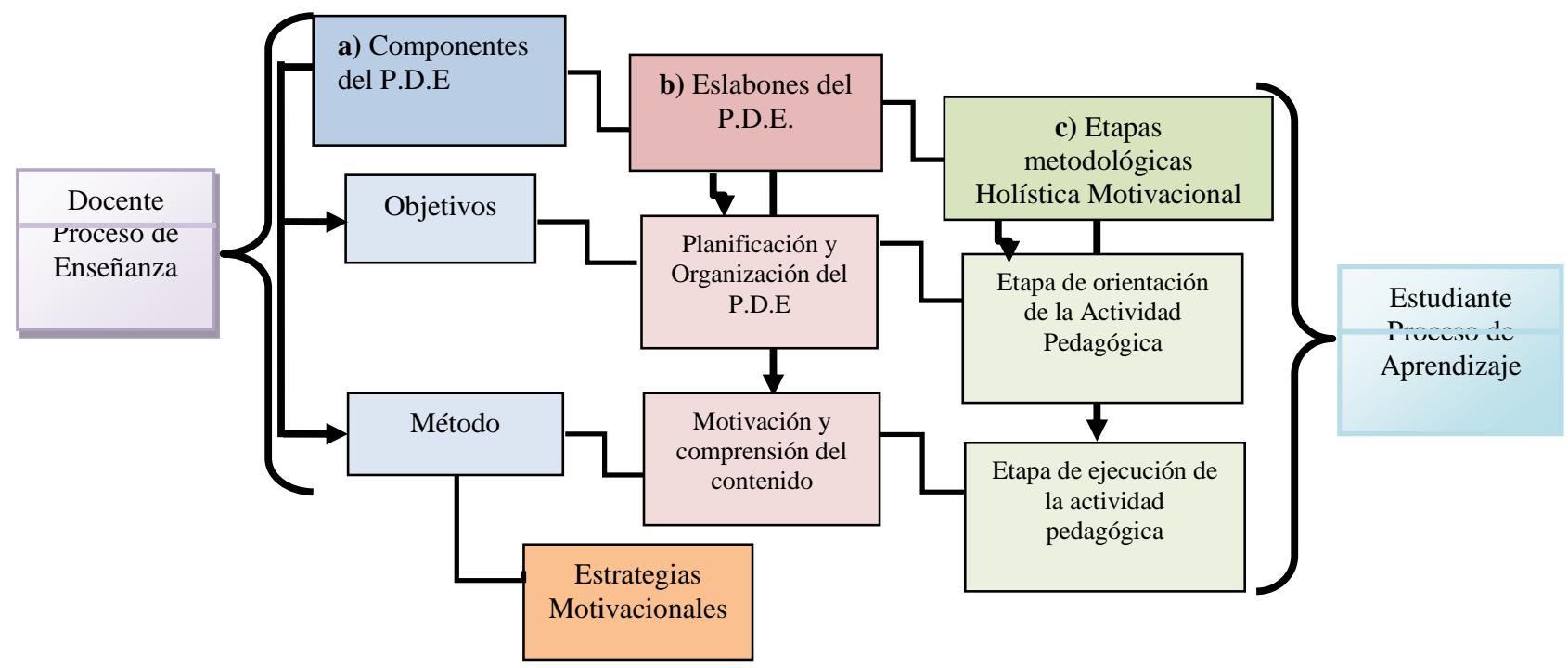

Figura 3. Desarrollo del modelo propuesto "métodos"

Los métodos de enseñanza y aprendizaje responden a las interrogantes: ¿Cómo desarrollar el proceso?, ¿cómo enseñar? y ¿cómo aprender? Están en directa relación con los restantes componentes del proceso y responden estrechamente a los objetivos y al tipo de contenido.

La metodología asumida en la asignatura será activa con métodos productivos, aunque se acepta la exposición, según la necesidad del tema a desarrollar el docente podrá organizar la forma de desarrollar los temas, en general y por la naturaleza de la asignatura. Las clases se desarrollaran mediante exposiciones basadas en análisis individual, participativo y de trabajo en grupo. Se organizarán grupos para investigar temas, exponerlos, etc., integrados por afinidad. 


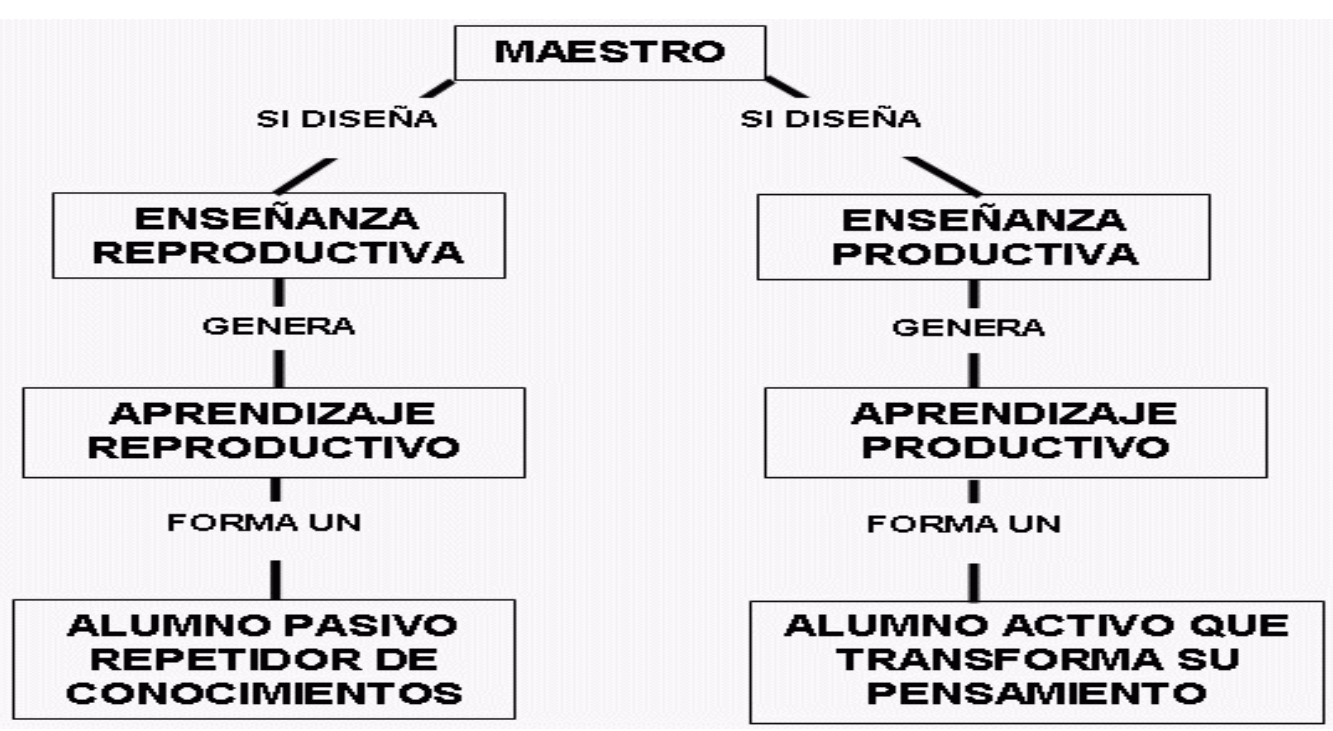

Figura 4. Método enseñanza productiva

Otro medio que el docente puede utilizar para mantener al estudiante atento $\mathrm{y}$ motivado es la televisión, ya que a través de imágenes y sonidos se persigue influir en el conocimiento, actitudes y valores en los espectadores a través de contenidos pedagógicos y didácticos, promoviendo la atención y a realizar juicios críticos de lo que se ve y lo que se escucha. De tal manera una vez concluida la presentación en tv como medio didáctico el grupo de estudiantes puede iniciar los debates, discusiones, juicios, etc. Dando lugar al método productivo por este medio. Por tanto los medios cumplirán las siguientes funciones:

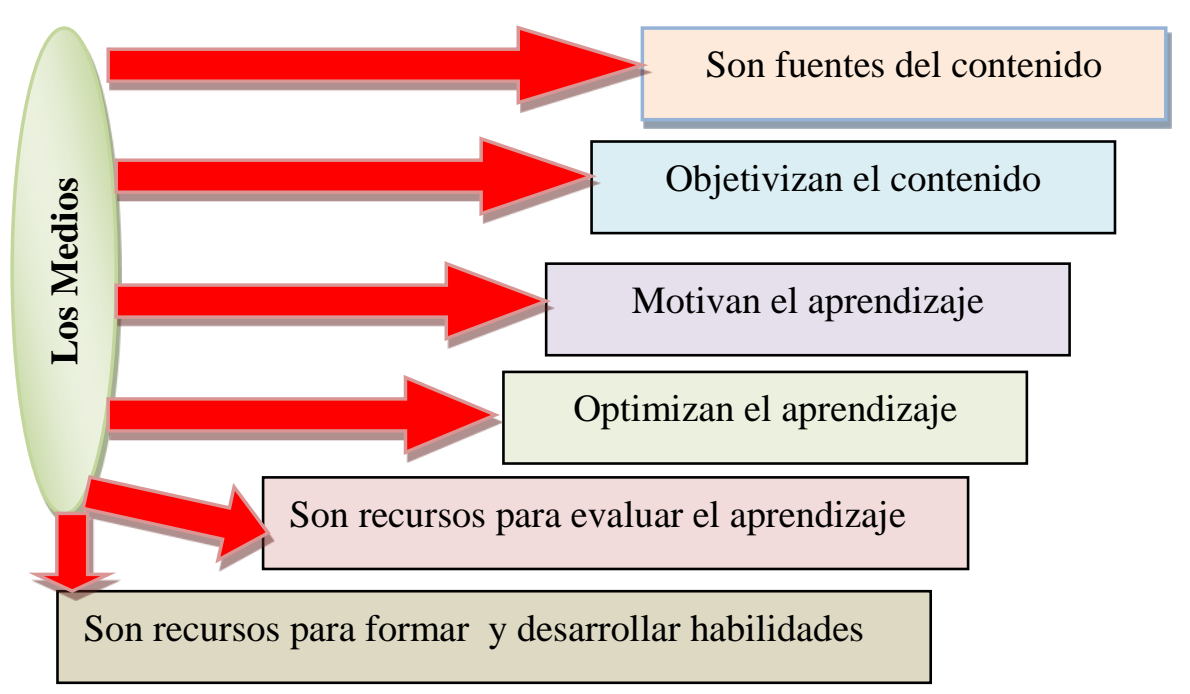

Figura 5. Funciones de los medios en la asignatura

Por lo que el uso de los medios propuestos es muy importante para la 
asignatura así mantener activa la participación de los estudiantes y brindar una mejor retención del aprendizaje, brindar mayor dinamismo en las clases, además de que permite la comunicación entre docentes y estudiantes.

Como se menciona en la teoría, la evaluación está a lo largo del proceso constatando el logro del objetivo por parte del estudiante, en vista de que en la asignatura se evalúa en 3 parciales, con exámenes en cada parcial y como se propone tener un método productivo de discusión plenaria, con estrategias motivadoras y con medios de interacción actuales que faciliten llegar al estudiante en cualquier momento y lugar, por lo que se requiere de nuevas formas de evaluar, por lo que además de la tradicional prueba individual, que se efectúa al estudiante de la asignatura, se propone efectuar pruebas a los grupos de trabajo de discusión plenaria.

Esta etapa de evaluación encontró gran significado para los alumnos en la medida en que los mismos valoren las dificultades a las que se enfrentaron, los compromisos que necesariamente se asumirán; clarificación de los problemas profesionales, de las expectativas y de los objetivos propuestos, así como de las perspectivas vinculadas con el propio proceso.

Es por ello, que la evaluación participativa posibilita la primacía de los valores humanos y el compromiso por la actividad profesional, porque permite realizar una valoración que tome en cuenta posibilidades de cambio y también limitaciones, que se insista en que se cambie lo que con actividades profesionales pueden ser cambiados.

En este sentido, se evaluó las dimensiones cognoscitivas y afectivas, así como las psicomotoras de los alumnos, de tal forma que permita la autonomía de las decisiones profesionales $\sin$ dañar la valoración de sí mismo.
La etapa de evaluación de la actividad comprenderá las siguientes acciones:

- Analizar la capacidad de los estudiantes en el proceso docente.

- Interpretar de forma general el cumplimiento de la actividad.(logros y dificultades)

- Valorar criterios para las diferentes variantes de actuación profesional.

La metodología propuesta no sólo se relaciona dialécticamente los referentes sociales y psicológicos del proceso docente educativo, sino los contenidos en su expresión compleja: conocimientos, habilidades, actitudes - valores. Esta no se identifica con el contenido (lo que el alumno debe aprender), o con los métodos (cómo el alumno aprenderá), sino con las características que alcanzará el estudiante cuando termine su aprendizaje. Es decir, la metodología supone transformaciones en el desempeño profesional, los cambios cualitativos, que se operan en el estudiante como producto de su actividad.

\section{Discusión}

Según Sosa (2017) una institución docente se proyecta en la sociedad, con el encargo de educar al hombre para la vida a partir de compromisos sociales, debiendo ser capaz de enfrentarse a nuevas situaciones y problemas que se le presenten y resolverlos en pos de transformar la sociedad. Es la integración holística y sistémica de la enseñanza y el aprendizaje de todos sus componentes junto con las cualidades, niveles de asimilación, de profundidad y estructural, en sus tres dimensiones: educativa, instructiva y desarrolladora. 
Por otra parte, Molto (1990) indica que el proceso Docente Educativo "es la actuación interrelacionada de los profesores $\mathrm{y}$ estudiantes, de estos últimos entre sí, de todos son determinados objetos para cumplir determinados objetivos, previamente definidos. A estos objetos se les denomina medios educativos.

Realizando un análisis de las definiciones sobre el proceso docente educativo, se observa que los autores concuerdan en señalar que dicho proceso debe estar enfocado a la formación, instrucción y educación, con el objetivo de preparar al estudiante para la vida, con criterio científico y creativo, mediante una estrecha relación entre estudiante y profesor, para que las nuevas generaciones den respuestas a los problemas del encargo social.

Lo cual es posible lograrlo mediante la aplicación sistemática de los componentes que caracterizan el proceso docente educativo: los objetivos, el contenido, el método, la forma u organización, el medio y la evaluación; a través de una estrategia de enseñanza que promueva la combinación de métodos y técnicas.

Sin embargo, Molto (ob.cit) manifiesta que en el proceso Docente - Educativo, además del profesor, los estudiantes, los objetivos, el contenido y los medios educativos existen los componentes siguientes: los métodos educativos, la superación, la evaluación y la retroalimentación, para este autor los objetivos constituyen la precisión del contenido de la profesión, en donde siempre están presentes intereses individuales, pero ellos a su vez responden a un determinado encargo social, de aquí la importancia de la conjugación de ambos aspectos.

Sosa (2017) menciona que el objetivo constituye aquel aspecto que mejor refleja el carácter social de éste y orienta la aspiración de la sociedad, es la imagen que se pretende formar de acuerdo con el encargo social planteado a la escuela; cumplir con este propósito resulta posible si se tienen en cuenta, además, los métodos empleados en este empeño.

Por otra parte, Ruiz y Fabregat (2008), señalan que los objetivos cumplen las siguientes funciones: una función rectora del proceso docente, una función orientadora y una función valorativa. Asimismo señalan que, los objetivos del Proceso Docente Educativo se elabora en diferentes niveles: los objetivos más generales de la formación profesional de un egresado, los objetivos de las disciplinas y asignaturas que conforman el plan de estudios y los objetivos para otros niveles más específicos. Por otra parte plantean que el método permite señalar que es el medio de alcanzar los objetivos y que los estudiantes se apropien del contenido, considerándolo como una de las características del proceso, que establece la lógica, el orden, la secuencia en la dinámica del proceso para arribar al fin.

Para Labarrete (2009) los procedimientos didácticos se encuentran en unidad dialéctica con los métodos, son un detalle de estos y complementan la forma de asimilación de los conocimientos que presupone determinado método; entre estos se encuentran: los dramatizados, la elaboración de resúmenes, los cuadros sinópticos, los esquemas lógicos, la utilización de software educativo, la consulta de fuentes bibliográficas, etc. Existen múltiples clasificaciones de los métodos de enseñanza - aprendizaje que se basan en criterios diferentes. Esta autora plantea, como los criterios más conocidos, los siguientes: por la fuente de adquisición de los conocimientos, y por la relación de la actividad del profesor y de los estudiantes.

Entonces, es necesario que los profesores se preparen en el manejo de un sistema de métodos activos, para explotar al máximo las 
potencialidades que ofrecen los mismos, para llevar adelante la difícil tarea de educar a través de la instrucción y recordar que la selección debe estar relacionada con el cumplimiento de los objetivos.

En tal sentido debe entenderse que el método es el modo de desarrollar el proceso en su estructura interna, es el componente que lo expresa en sí mismo, donde se manifiesta su carácter fenoménico, mientras el objetivo es su esencia, el método es el fenómeno. Los objetivos están determinados por las necesidades y exigencias sociales dadas en el marco de la escuela, el tipo de enseñanza, la asignatura y el grado.

Ahora bien, los contenidos, por su parte, materializan los conceptos, leyes, principios y teorías que sirven de base a los objetivos planteados. Representan el "qué" se enseña; los contenidos no solo tienen un carácter informativo sino que en ellos están presentes elementos que contribuyen a la formación de convicciones, a la educación general del estudiante, y que le sirven de soporte a los procesos de establecer ciertos algoritmos que a su vez facilitan la formación de hábitos y habilidades.

El cumplimiento de los objetivos solo se hace posible mediante el método de enseñanza que establece la secuencia que el profesor desarrolla para lograr sus propósitos educativos, instructivos y desarrolladores; responde al "cómo", es decir, a la manera de actuar para lograr lo propuesto. Según el método empleado se decidirá en buena medida el tipo de medios a utilizar, estos responden al "con qué" enseñamos.

De la misma manera los medios, se denominan medios de enseñanza a todos los medios materiales que necesitan profesores y estudiantes para una estructuración efectiva y racional. Los medios permiten crear las condiciones favorables para cumplir con las exigencias científicas del modelo pedagógico.
Determinados el objetivo, el contenido, los métodos y los medios y respondidas con ellos las interrogantes: "para qué", "qué", "cómo" y "con qué", no cabe dudas de que procede entonces, el " dónde y cuándo", preguntas que encuentran respuestas en el más dinámico de los componentes del proceso: la forma de organización docente.

\section{CONCLUSIONES}

El presente trabajo fue desarrollado en la asignatura de Administración de la Producción III de la carrera de Administración de Empresas de la Universidad Autónoma "Tomás Frías".

La Forma de Organización Holística Motivacional estriba en su capacidad de estimular conscientemente al estudiante; La motivación dentro del proceso enseñanza y aprendizaje, es un sistema abierto, flexible y autorregulado, tanto pedagógicas como no, siendo estimulado para lograr los objetivos que se pretenden.

El diagnóstico realizado en base a los instrumentos aplicados a los estudiantes y docentes de la asignatura de Administración de la Producción III permitió manifestar a los estudiantes su preocupación y sugerencias para mejorar la materia.

El modelo holístico motivacional propuesto dirigido a mejorar el proceso enseñanza y aprendizaje, en la asignatura de Administración de la Producción III de la carrera de Administración de Empresas de la Universidad Autónoma "Tomás Frías" y tomando en consideración los preceptos teóricos asumidos, aporta una metodología al docente para motivar a los estudiantes, que es viable.

El modelo puede ser aplicado para poder mejorar el proceso enseñanza y aprendizaje en la asignatura de la Administración de la Producción III, de la carrera de Administración de Empresas de la Universidad Autónoma “Tomás Frías". Logrando así que las clases sean 
participativas, motivacionales, activas, de debate, que interese al estudiante.

Además, es muy importante que en todas las materias de la carrera pueda existir una relación de teoría y práctica, fortaleciendo el proceso enseñanza y aprendizaje con miras a la futura profesionalización de los estudiantes.

\section{REFERENCIAS}

Álvarez, M (2006). Los componentes del proceso docente educativo. La Habana: Editorial Academia

Labarrete, G (2009). Pedagogía. La Habana: Editorial Pueblo y Educación

Molto, E. (1990) El Diseño del Proceso Docente Educativo en la Educación Superior tesis en opción al grado científico de Doctor en Ciencias Pedagógicas. Cuba Universidad de la Habana

Ruiz, H. y Fabregat, P. (2008) El Proceso Docente Educativo La Habana, Editorial El Fuego,

Ruíz, D. (2015) Artículos de reflexión revista contexto social Bogotá Colombia Programa de Trabajo Social, Escuela de Ciencias Humanas y Sociales Fundación universitaria Monserrate Recuperado de http://www.unimonserrate.edu.co/wpcontent/uploads/2018/04/2.1.CRITERIO S-ART\%C3\%8DCULOS.pdf

Sosa, M. (2017) El proceso docente educativo: Sus componentes. Argentina Edición Lid Editor 